\title{
Secretory activity of mandibular organ fluctuates in response to reproductive season of the field crab Paratelphusa sp. (Brachyura; Decapoda): an ultrastructural study
}

SN Sarika', N Gayathri ${ }^{2}$ and Anilkumar Gopinathan ${ }^{1 *}$

\begin{abstract}
Background: Mandibular organ (MO) in decapods is suggested to play regulatory role in reproduction, in few species; however, MO is considered to control growth. The present study addresses this question by an ultrastructural study on the MO of the field crab, Paratelphusa sp. Our sampling for consecutive years (2008 to 2012) revealed that Paratelphusa sp. devotes July to October for reproduction, judged by the occurrence of growing ovaries and the berried females. From November to the succeeding June, the females are in a state of reproductive arrest (non-reproductive period); ovaries during this season would appear as white bands with no signs of yolk deposition.
\end{abstract}

Results: Morphologically, MO of Paratelphusa sp. is positioned posterior to the mandibles and is in close apposition with the distal end of the mandibular apodeme. MO of Paratelphusa sp. exhibited significant levels $(t=8.097$, $P<0.0001, N=10$ ) of season-dependent size variations. Our electron microscopic observations reveal that the $\mathrm{MO}$ is highly secretory during the reproductive period, evidenced by the occurrence of sacculated Golgi bodies having dense inclusions, several mitochondria with tubular cristae, and extensive networks of SER and rough endoplasmic reticulum (RER). During the non-reproductive period, however, the MO is least active; RER, the mitochondria, and the Golgi are only sparsely seen. Interestingly, the plasma membrane exhibits a highly convoluted appearance all the way through the non-reproductive period.

Conclusions: The present study reveals that the secretory activity of $\mathrm{MO}$ of Paratelphusa sp. is entrained with reproductive activity. The existence of a high correlation between $\mathrm{MO}$ secretory activity and ovarian growth implicates the former's role in reproduction.

Keywords: Mandibular organ; Reproduction; Brachyuran crabs; Decapoda; Paratelphusa

\section{Background}

The mandibular organ (MO) in crustaceans is a pair of ductless, ectodermally derived gland, first described by Le Roux (1968) in the cephalothorax of the crab Carcinus maenas. Initial studies by several authors suggested the MO to be involved in regulating reproduction (Le Roux 1968. Hinsch 1981), and/or molting (Aoto et al. 1974, Byard et al. 1975, Yudin et al. 1980). A major limitation in

\footnotetext{
* Correspondence: ganilkumar@vit.ac.in

'School of Bio Sciences and Technology (SBST), VIT University, Vellore, Tamil Nadu 632014, India

Full list of author information is available at the end of the article
}

investigating the exact role of MO has been its anatomical location which has made its surgical removal difficult. Therefore, it has not been possible to perform the 'classical' ablation-replacement experiments to probe into the MO function, unlike the situation in the corpora allata of insects (Nagaraju et al. 2006).

In the 1990s, studies on MO were conducted in the context of changes in the nature of its secretion, a terpenoid compound, farnesoic acid (FA), a pro-hormone that gets methylated by farnesoic acid O-methyl transferase to the active form of the hormone methyl farnesoate (MF) (Gunawardene et al. 2002). The physiological 
function for MF was enigmatic in crustaceans until its analogy with the gonadotropic hormone JH III from insects was established (Laufer et al. 1987, Borst et al. 1987); a recent report suggests MF to be the precursor for insect $\mathrm{JH}$ (Teal et al. 2014). In vitro and in vivo experiments have demonstrated an association between MF production and ovarian development in C. maenas, Libinia emarginata, and Procambarus clarkii (Borst et al. 1987, Laufer et al. 1998, Nagaraju et al. 2006, Nagaraju 2011). Recent studies on the biosynthesis and release of farnesoic acid and MF within the gland (MO) revealed that the rates of biosynthesis of FA and MF were stagedependent, with maximal production (with a threefold increase) occurring during secondary vitellogenesis, i.e., stages 4 and 5 in Homarus americanus (Tiu et al. 2012). Since the discovery of MF in crustaceans, the physiological role of this compound has been a subject of debate. While some of the authors are of the view that MF could be stimulating molt (Yudin et al. 1980, Tamone and Chang 1993), there have been suggestions that MF could play a crucial role in delaying the onset of molting in larval crustaceans (Borst et al. 1987).

Molting and breeding are seasonal events in crustaceans. Most of the brachyuran crabs program their molt (premolt growth) and breeding in antagonistic fashion; only the intermolt females engage in breeding (Adiyodi and Adiyodi 1970, Sudha and Anilkumar 2007, Kappalli et al. 2012). However, any questions on the regulatory mechanisms of these seasonal events could be addressed effectively only if the studies are conducted in a seasondependent fashion; and a season-wise study on the MO secretory activity is totally lacking in brachyuran crustaceans. It is, at this juncture, we undertook the present study that depicts the results of ultrastructural study on the MO activity of Vellore population of the field crab Paratelphusa sp. during the reproductive and the nonreproductive seasons from July to September and March to June/July, respectively.

\section{Methods}

\section{Collection and maintenance of animals}

All adult female crabs (within the size class of 3.2 to $3.8 \mathrm{~cm}$ carapace width) used for the present study were in intermolt and were collected from rice farms and adjacent areas of the VIT University campus, Vellore, India $\left(12.56^{\circ} \mathrm{N}, 79.8^{\circ} \mathrm{E}\right)$. Collections were made by baiting, on a weekly basis; 35 to 40 animals were observed each month to assess the impact (if any) of seasonal variation in breeding and molting. In the laboratory, the crabs were reared in plastic cisterns and were fed $a d l i b$ on boiled beef liver, boiled rice, and tender grass (after Sudha and Anilkumar 2007; Kappalli et al. 2012). Adequate care was taken to maintain them in near-natural condition. Special care was taken to clean the cisterns regularly to remove the leftover food materials so as to minimize or avoid stress to the animals, conforming to the International guidelines for maintenance of laboratory animals (NRC, 2011, Guide for the Care and Use of Laboratory Animals, Eighth Edition).

\section{Identification of stages of vitellogenesis and molting}

The ovary was dissected out, by cutting open the dorsal part of the carapace; the dissection was conducted in normal physiological saline medium. For characterization of the vitellogenic stages, oocyte samples were placed on a temporary wet-mount microscope slide and their dimensions were measured using a calibrated ocular micrometer, to the nearest one micrometer. Taking the oocyte size, the ovary wet weight, and the color of the ovary as the criteria, vitellogenesis of Paratelphusa sp. is classified into four stages (1 to 4 ). The ovary at stage 1 of vitellogenesis appears creamy with oocyte diameter ranging between 300 and $500 \mu \mathrm{m}$. Stage 2 ovary is bright yellow, with the oocyte diameter ranging between 501 and $800 \mu \mathrm{m}$ (Figure 1a). The ovary attains a brown or brownish black hue as vitellogenesis progresses towards stages 3 and 4 ,

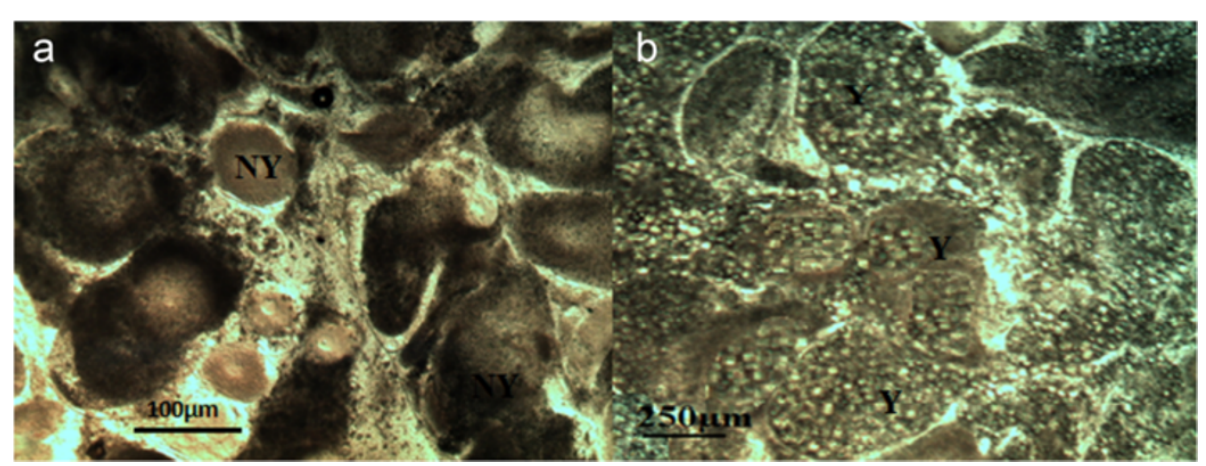

Figure 1 Non-yolky and yolky oocytes during non-reproductive and reproductive seasons. (a) shows non-yolky oocytes, NY (non-reproductive season). (b) shows yolky oocytes (Y) usually occur during secondary vitellogenesis (reproductive season). 
the oocyte diameters being 801 to 1,000 and 1,001 to $1,440 \mu \mathrm{m}$, respectively (Figure $1 \mathrm{~b}$ ).

Molt stages were identified by microscopic observations of the setogenic events in the pleopods and through macroscopic observation of changes in the exoskeleton. The tip of a pleopod was cut out using a pair of scissors, mounted in water on a clean glass slide, and observed light microscopically. Morphological characteristics of setae on the pleopods and the extent of epidermal retraction were used to define the stages of molting: premolt, the stage preparatory to molting, is classified into substages D1 to D4 which are characterized by stages of setogenic events identified clearly and precisely through a light microscope. A clear but narrow margin between the epidermal layer and the cuticle defines stage D1. A wider margin of the retracted epidermal layer can be seen in stage D2, followed by appearance of rudiments of (juvenile) setal grooves (stage D3), and subsequently by the occurrence of fully developed juvenile setae (D4), ready to replace the old set of setae. Soon after exuviation, the animal possesses a soft exoskeleton. The newly formed setae at this stage (A) do not possess the setal cones; at stage B, the setal cones start appearing. Subsequently, in another 5 to 7 days, the crab transforms into the intermolt stage.

\section{Dissection of mandibular organ}

The mandibular organ of Paratelphusa sp. was dissected out by cutting open the carapace from the area adjacent to the chitinous apodeme projecting outwards from the dorsal cavity. After having dissected out, the tissue was immediately fixed in $10 \%$ neutral buffered formalin for histological studies and in 3\% buffered glutaraldehyde for electron microscopic studies.

\section{Histology}

For histological examinations, the tissue, fixed in 10\% neutral buffered formalin, was dehydrated in a graded alcohol series and cleared in xylene for $15 \mathrm{~min}$. The tissues were then embedded in Paraffin wax, sectioned at 5- to 7- $\mu \mathrm{m}$ thickness, and stained with hematoxylin and eosin for light microscopic observations.

\section{Transmission electron microscopy}

The tissue, after being fixed in 3\% buffered glutaraldehyde for $24 \mathrm{~h}$, was washed thoroughly with $0.1-\mathrm{M}$ phosphate buffer and post-fixed in osmium tetroxide for 1 to $2 \mathrm{~h}$ at $4^{\circ} \mathrm{C}$. After a brief wash in 0.1-M phosphate buffer, it was dehydrated in graded series of ethanol $(70 \%$ to $90 \%)$. Following dehydration in $90 \%$ ethanol, the sample was incubated in (freshly prepared) $2 \%$ ethanolic uranyl acetate (en bloc staining) and was subsequently dehydrated with $100 \%$ ethanol. Propylene oxide was used as the clearing agent. Further, the tissue was left for infiltration for a minimum of $6 \mathrm{~h}$ in a 1:1 mixture of propylene oxide and araldite followed by pure araldite for a further period of $8 \mathrm{~h}$. The tissue was then embedded in araldite and kept in an oven maintained at $60^{\circ} \mathrm{C}$ undisturbed for 2 days for polymerization. Semi-thin sections $(1 \mu \mathrm{m})$, stained with $1 \%$ toluidine blue, were used to ensure the exact positioning of the tissue. Ultra-thin (600 $\mathrm{A})$ sections contrasted with uranyl acetate and lead citrate were scanned under FEI TECNAI Biotwin electron microscope (FEI, Hillsboro, OR, USA).

\section{Results}

Seasonal changes in physiology of Paratelphusa sp. with reference to molt and reproduction

The physiology of Paratelphusa sp. was examined throughout the year in a season-dependent manner with special reference to molt and reproduction. Our sampling from the year 2008 to 2012 revealed that the seasonal physiological cycle of the species could be broadly classified into two seasons: reproductive season and non-reproductive season. Reproductive season begins with the onset of monsoons from July onwards, the adult female crabs showed signs of developing ovary and as the monsoon progressed (August to September), the ovaries showed signs of yolk deposition. At the end of September and early October, egg-carrying females as well as juveniles were visible in the fields. This was followed by the frequent molts of the juveniles until the end of November. From November onwards, the animals appear to prepare for their pubertal molt. Until February and early March, the entire population appeared to be engaged in molting; occurrence of various postmolt (with a soft exoskeleton) and intermolt organisms appear by the end of March. The nonreproductive season (a state of ovarian repose) begins from March, where the ovaries at this stage would appear as white bands with no signs of yolk deposition. Subsequently, during March to June, there is a complete inactivity in the population; the females engage neither in reproduction nor in molting.

\section{Localization and gross morphology of mandibular organ} Mandibular organ of the field crab Paratelphusa sp. is a pair of pale yellow, somewhat spherical, and is approximately 1 to $1.5 \mathrm{~mm}$ in diameter. $\mathrm{MO}$ is seen directly posterior to the mandibles and closely associated with the anterior portion of the paired chitinous tendons that extend from the mandibles to the dorsal carapace. Layers of connective tissue surround and firmly attach the MO to the tendons (Figure 2).

\section{Histology of MO}

The histological sections of $\mathrm{MO}$ reveal that the glandular epithelium is surrounded by an outer connective tissue 


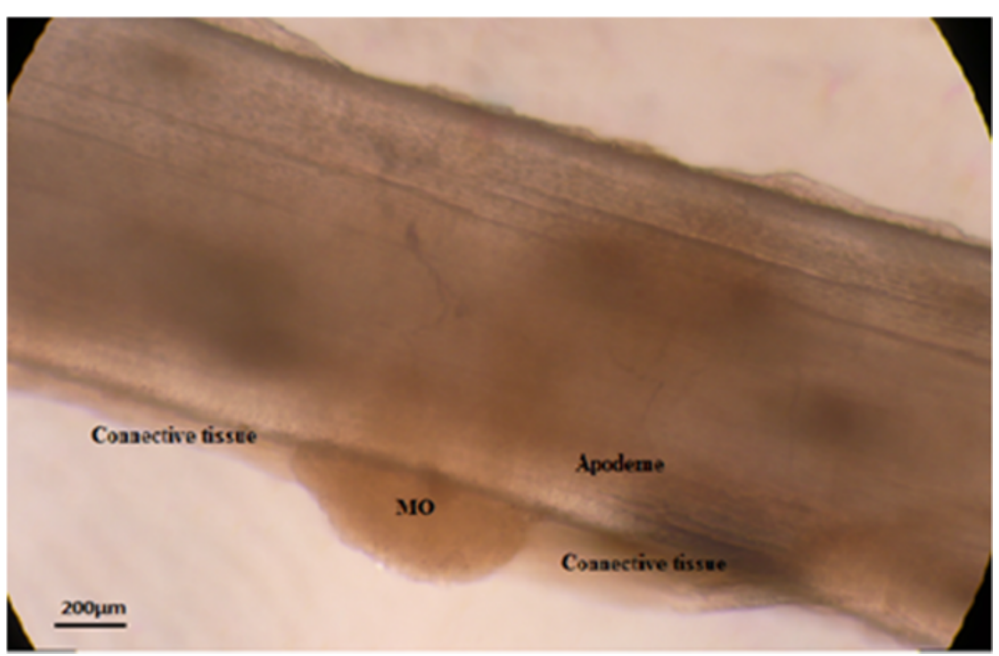

Figure 2 A typical mandibular organ (MO) of Paratelphusa sp. attached to the apodeme from reproductive period.

followed by an inner muscle layer which helps firm attachment of the gland to the apodeme (Figure 3).

\section{Ultrastructure of MO}

Ultrastructurally, the cord configuration of the mandibular organ is clearly demonstrated as seen in Figure 4. Each cell is surrounded by intercellular hemolymph channels which in turn are interconnected with hemolymph sinuses. Granular and agranular hemocytes were noted within the sinuses (Figure 5a,b,c). Granular hemocytes characterized by irregularly shaped nuclei were usually found outside the sinuses. On the other hand, agranular hemocytes are restricted within the sinuses. The mandibular gland cells vary in shape and tend to have eccentric nuclei with condensed heterochromatin around the peripheral karyoplasm.

\section{Season-dependent morphological changes in $\mathrm{MO}$}

MO of Paratelphusa sp. exhibited significant levels $(t=$ 8.097. $P<0.0001, N=10$ ) of season-dependent size variations. The gland was found to be pale-yellow and somewhat spherical, with size ranging from $0.629 \pm 0.072 \mathrm{~mm}$ during the non-reproductive season to $1.015 \pm 0.078 \mathrm{~mm}$ during the reproductive season.

\section{Season-dependent ultrastructural changes of MO Non-reproductive season (March to June/July)}

During the months of March to June, each cell appears to be surrounded by a few or more granular hemocytes within the adjoining hemolymph channels. The plasma membrane of mandibular gland cells during the season, exhibits profuse convolutions especially where it is adjacent to hemolymph channels (Figure 6a); SER, RER,

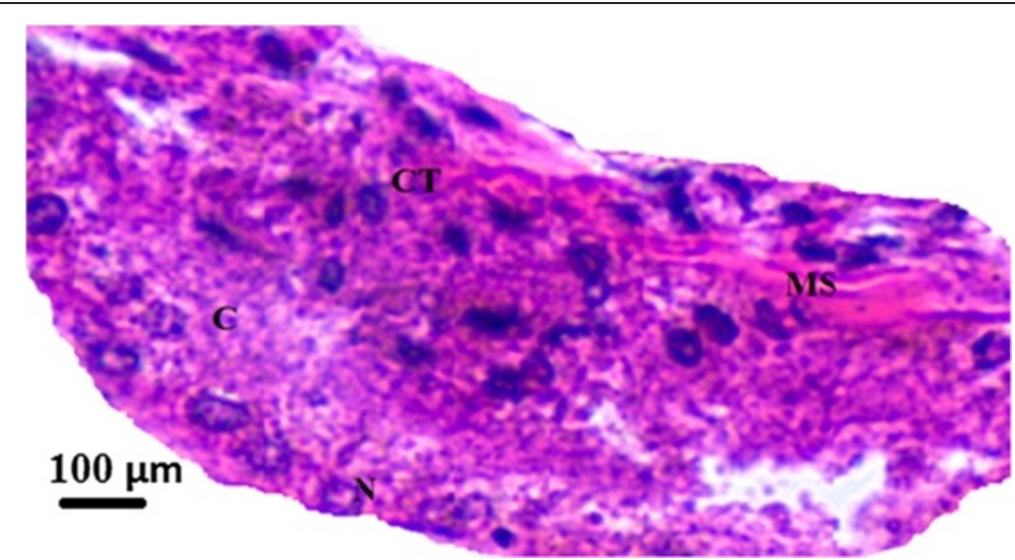

Figure 3 Light microscopic image of MO nucleus, cytoplasm and muscle. Light microscopic image depicting an overview of MO ( $N$ ) nucleus, (C) cytoplasm showing an outer ensemble of connective tissue (CT), and muscle (MS) from the reproductive period. 


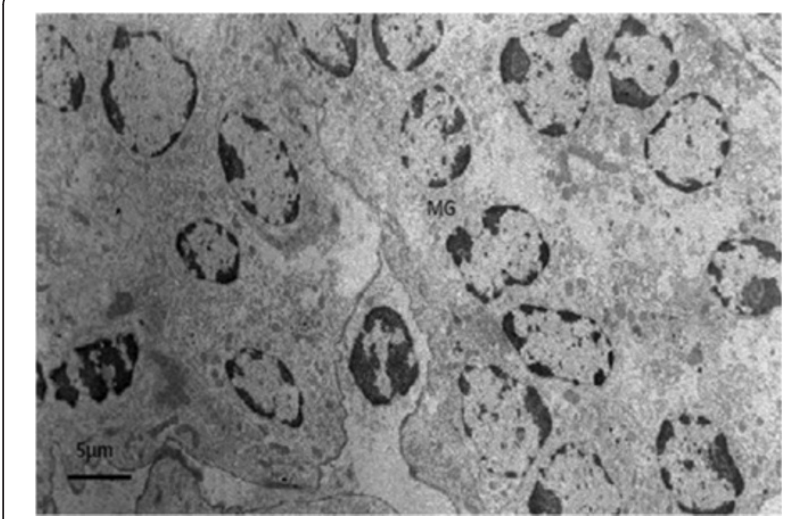

Figure 4 Mandibular gland cell cord like arrangement surrounded by hemolymph channels.

Golgi, and the mitochondria (Figure $6 \mathrm{~b}, \mathrm{c}$ ) are only rarely seen. Ribosomes are found scarcely scattered in the cell (Figure 6b).

\section{Reproductive season (July to September)}

During the months of July to September (premonsoon and monsoon seasons), MO of Paratelphusa sp. displays signs of high activity. A prominent feature during this period is the abundant appearance of granular and agranular hemocytes in and around the hemolymph sinuses (Figure 7a,b,c). Another feature of the cytoplasm of active mandibular gland cells is the presence of several dense mitochondria (Figure 8a). Golgi complexes are also dispersed throughout the cytoplasm, often with dense, lipidlike inclusions near their concave surfaces (Figure 8b). These inclusions usually are scattered throughout the cytoplasm. The Golgi complexes are frequently observed in close association with large accumulations of RER and SER (Figure 8c and Figure 9a,b). In such associations, the convex surface of the Golgi complexes appears to produce the saccules, and the concave surface releases the vesicles. The active mitochondria appear to be having tubular cristae oriented perpendicularly to their long axes with elaborate networks of smooth and rough endoplasmic reticulum dispersed throughout the cytoplasm within a minimally convoluted plasma membrane (Figure 10). Another prominent feature of a typically active mandibular gland cell is the occurrence of distinct electron dense cells rich in SER (Figure 11). They were found to be appearing between the gland cells and near the lining of the hemolymph sinusoid (Figure 12).

\section{Discussion}

This is the first ever report on the structure and function of MO of a brachyuran crab in a season-dependent manner. The histology and ultrastructure of mandibular organ have been examined through the entire annual cycle, involving the breeding (July to November) and the non-breeding (March to June) seasons of this field crab.

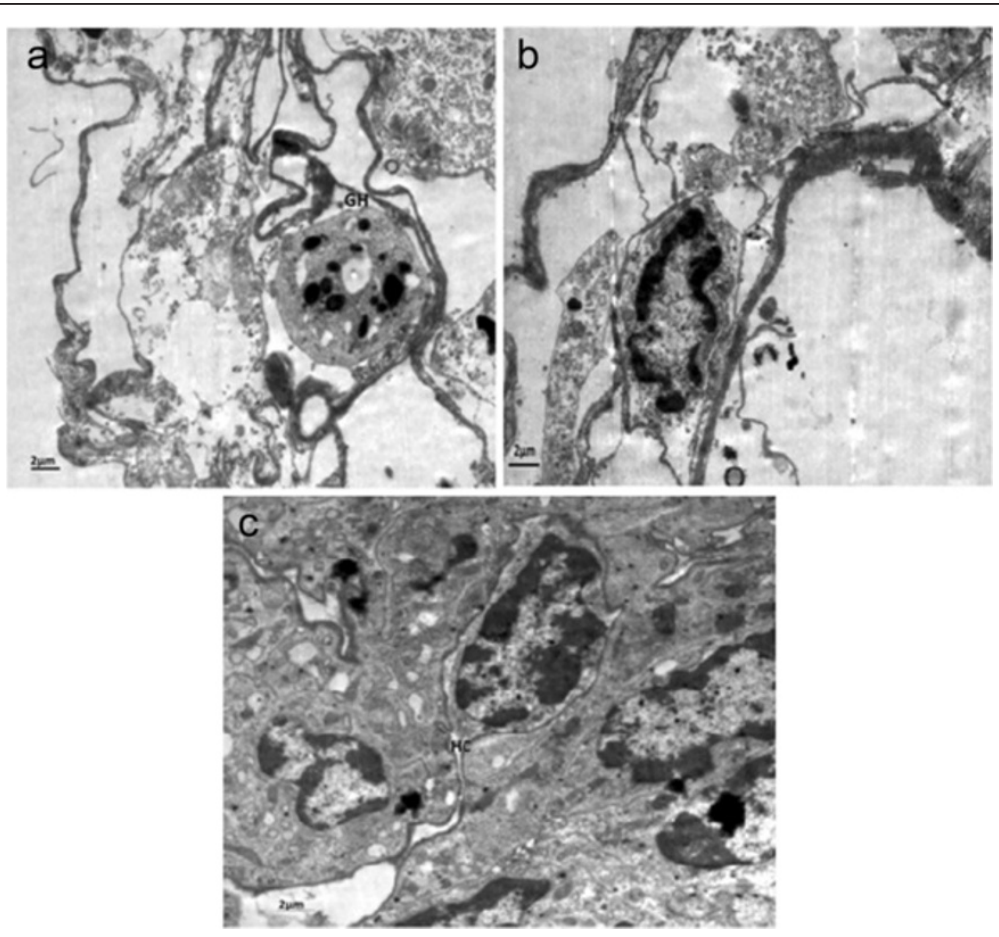

Figure 5 Granulated and agranular hemocytes and mandibular gland cell. (a) Granulated hemocyte (GH), (b) Agranular hemocyte within the sinusoid, and (c) mandibular gland cell surrounded by hemolymph channel (HC). 


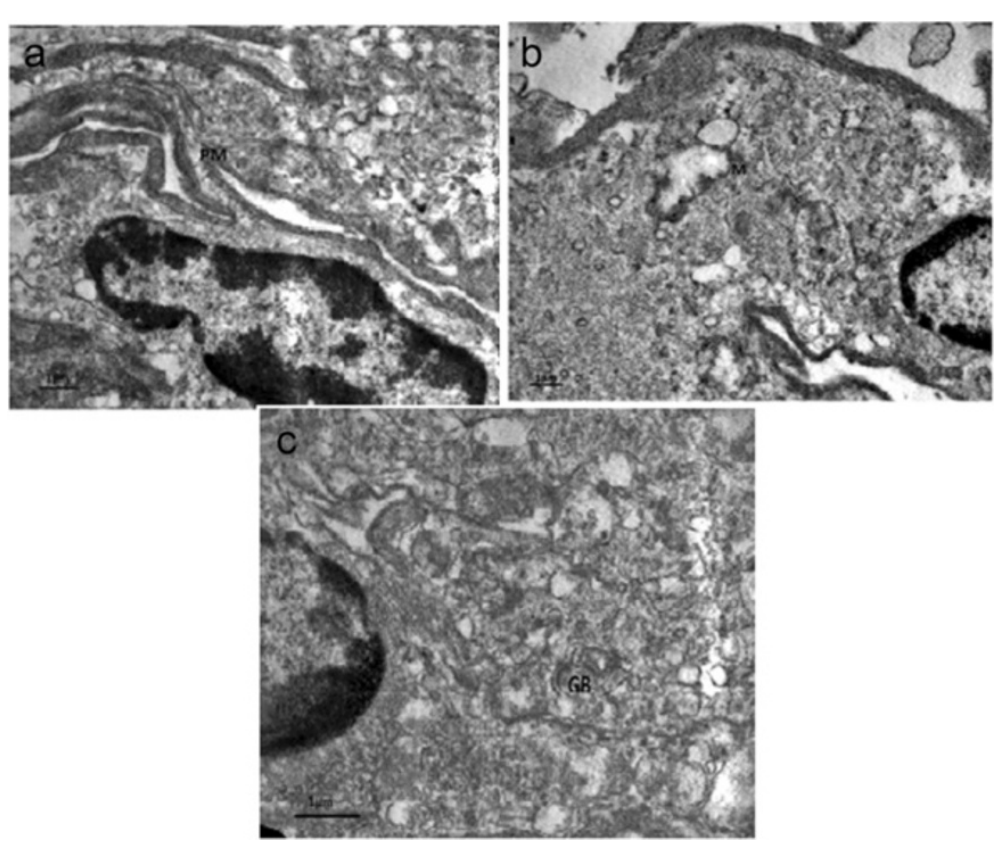

Figure 6 Ultrastructure of MO during non-reproductive season. (a) Plasma membrane convolutions, (b) mitochondrion, and (c) the Golgi complex.

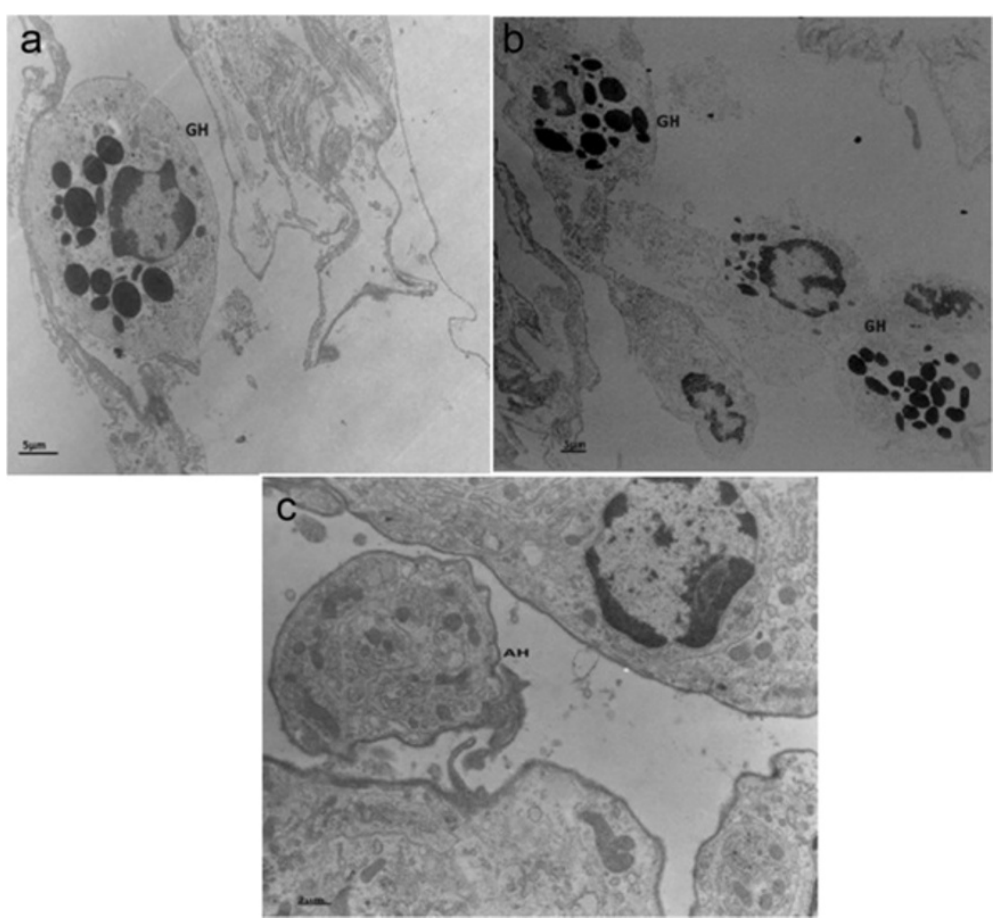

Figure 7 Granulated hemocytes and typical agranular hemocyte. (a) and (b) shows the granulated hemocytes in a typically active mandibular gland cell and (c) a typical agranular hemocyte in the sinusoid. 


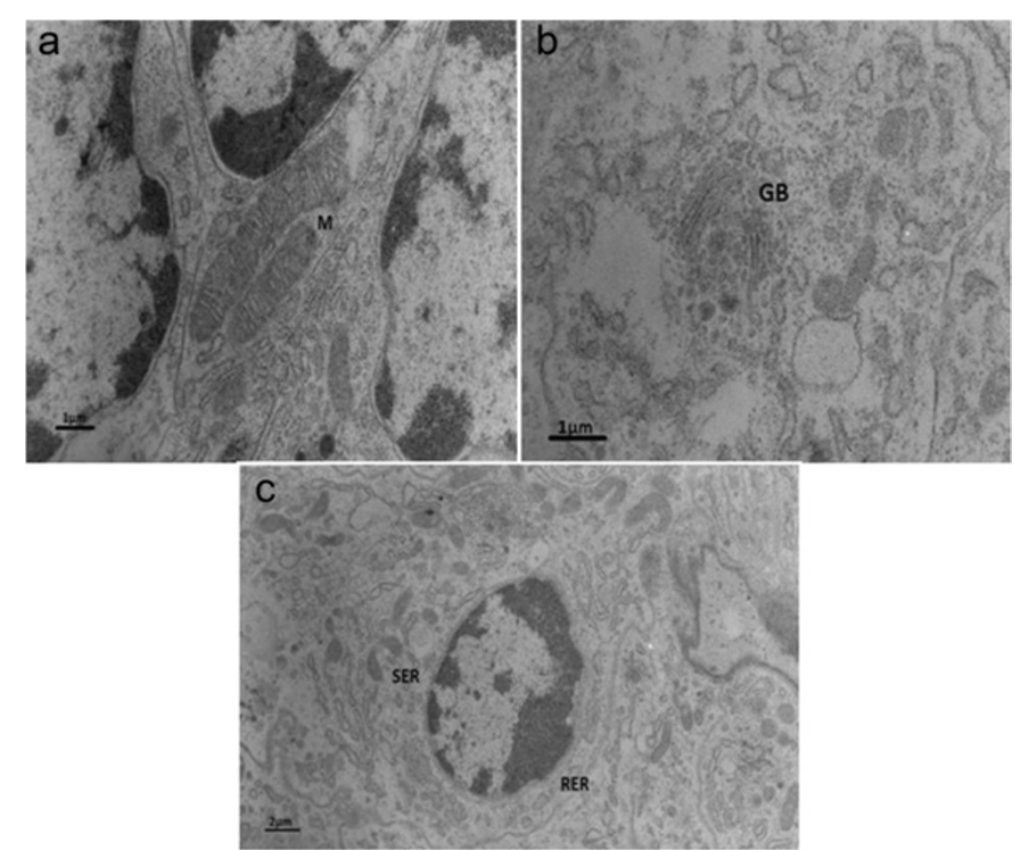

Figure 8 Mitochondrion, Golgi bodies, and mandibular gland cell. (a) Mitochondrion (M) of a typically active cell, (b) Golgi bodies (GB) with tubular cisternae and dense vesicles, and (c) mandibular gland cell cytoplasm with SER and RER.

MO attains maximum size during the months of July to November ( 1 to $1.5 \mathrm{~mm}$ ), the season where the entire population devotes itself for reproduction. The gland has its minimum size $(0.6 \mathrm{~mm})$ during the months of March to June, when the population does not engage in reproduction.

The histological and ultrastructural examinations of MO revealed that the mandibular gland cells of Paratelphusa sp. conformed closely to non-peptide secreting cells, judged by the occurrence of profuse SER and the Golgi (Figure 8c). However, these cells do not seem to be involved in steroid secretion, inasmuch as the $\mathrm{MO}$ is known to secrete the terpenoid compound such as the methyl farnesoate (Laufer et al. 1987). It would be pertinent to recall that the cellular nature of $\mathrm{MO}$ of the blue crab Callinectes sapidus has been suggested to resemble steroid secreting cells (Yudin et al. 1980). Another cytological feature of the MO cells of Paratelphusa sp. has been the occurrence of convolutions in the plasma membrane during non-reproductive season when compared with the reproductively active season. We are encouraged to presume that these convolutions could be the aftermath of inactivity during the non-breeding season (and the resultant decrease in the cell size). These fine structural details on the gland add to our understanding on its function and reiterate its active role in reproduction.

A variety of other cytoplasmic inclusions are seen in the MO cells during reproductive season. Some of them, with a fairly granular matrix of moderate electron density, are frequently observed to contain electron dense materials of various shapes. Other inclusions have a highly electron dense matrix bounded by a limiting membrane (Figure 11). None of these structures were observed during non-reproductive season. Further, the fine structural details of the organ showed a remarkable

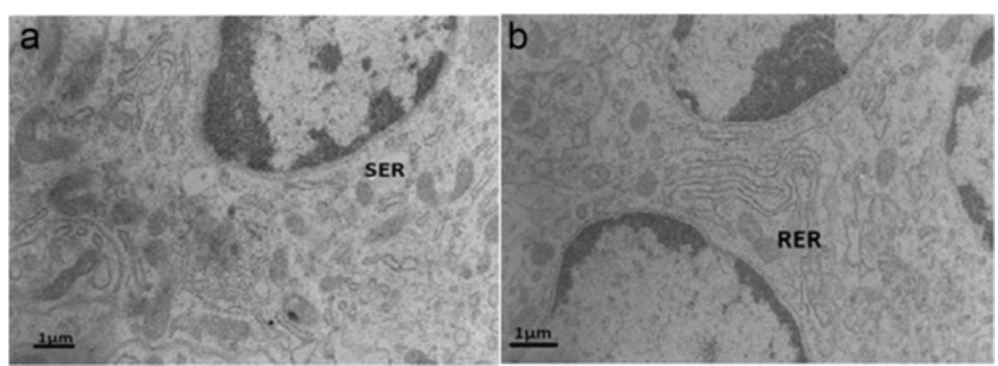

Figure 9 Higher magnification of SER (a) and stack of RER (b). 


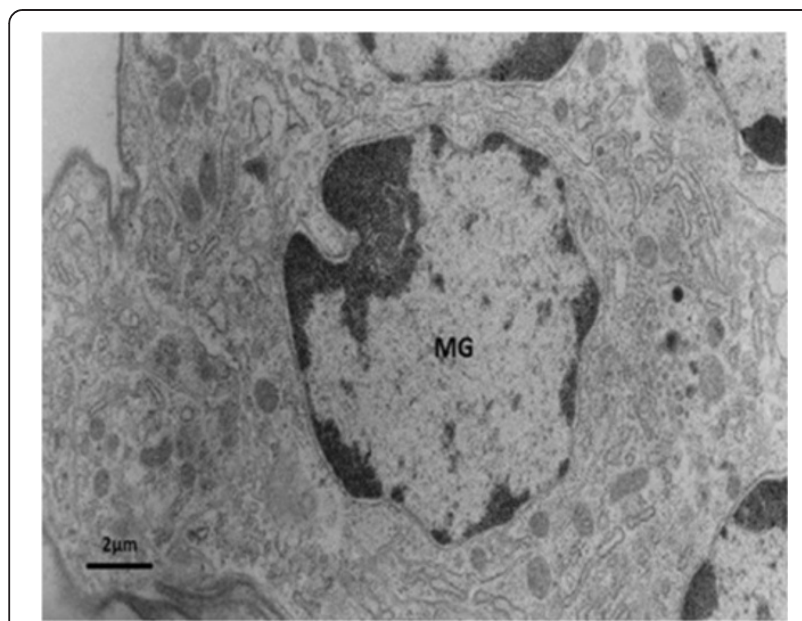

Figure 10 Enlarged image of an active cell of MO exhibiting smooth plasma membrane. difference in the occurrence of granular as well agranular hemocytes in the sinusoid (Figure 7a,b) between the reproductive and the non-reproductive seasons. The profuse occurrence of hemocytes during the reproductive season suggests for their role in transportation or storage of the secretory materials of the gland. With respect to the mode of release of the secretory materials, we failed to observe the signs of either apocrine or holocrine mode of secretion; presumably, the mode of release of the secretory product of $\mathrm{MO}$ could be merocrine.

Further, admittedly, it would be worth mentioning in this context that the exact signaling of MF leading to reproduction in crustaceans is still enigmatic. Moreover, the sequencing studies conducted of late in our laboratory demonstrated that the RXRDBD (accession number: JQ239171) of Paratelphusa sp. is remarkably identical (75\% to $78 \%$ identity) with the USP of Drosophila

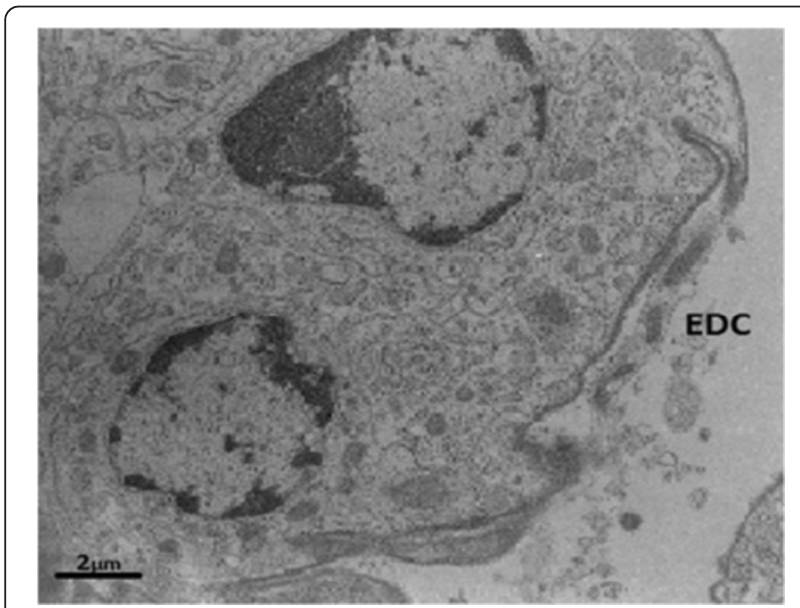

Figure 11 Distinct electron dense cells (EDC) lining the sinusoid.

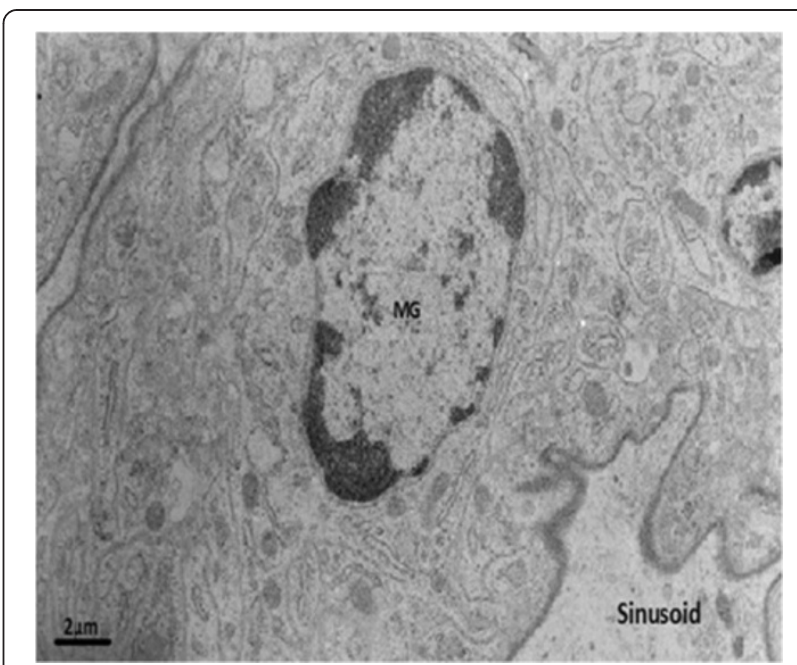

Figure 12 Gland cell with sinusoid on the side.

melanogaster, Aedes aegyptii, Bombyx mori, and Manduca sexta (Sarika and Anilkumar 2014). Pertinently, the observation of in vitro binding between sesquiterpenoids and retinoid receptor $(R X R)$ gene, leading to the latter's activation to elicit hormone response (Wang and LeBlanc 2009), has encouraged the endocrinologists to suggest RXR as a putative receptor for MF (Nagaraju et al. 2011, Tiu et al. 2012). Future experiments involving binding assays and expression studies (on a season-dependent fashion) would help us understand explicitly the exact mode of hormonal action for the successful accomplishment of reproduction in decapod crustaceans.

\section{Conclusions}

To conclude, the present ultrastructural study has demonstrated (for the first time) the fluctuating secretory activity of the mandibular organ of a brachyuran decapod (Paratelphusa sp.) in the context of reproduction, in a season-dependent manner. We consider this study as a prelude to our understanding on the mechanisms involved in the regulation of decapod reproduction. The present observation, along with those of Metapenaeus ensis demonstrating the synthesis of farnesoic acid by the MO (Gunawardene et al. 2002), and the fluctuating levels of MF in tune with the breeding activity in Oziotelphusa senex senex (Nagaraju et al. 2004) reinforces the suggested role of MO (and its secretion) in decapod reproduction.

\footnotetext{
Abbreviations

Y: yolky oocytes; NY: non-yolky oocytes; MO: mandibular organ;

MG: mandibular gland; N: nucleus; C: cytoplasm; CT: connective tissue; MS: muscle; GH: granulated hemocyte; AH: agranulated hemocyte; HC: hemolymph channel; M: mitochondrion; GB: Golgi bodies; SER: smooth endoplasmic reticulum; RER: rough endoplasmic reticulum; EDC: electron dense cell.
} 


\section{Competing interests}

The authors declare that they have no competing interests.

\section{Authors' contributions}

SSN and AG have made substantial contributions to the conception, design, and acquisition of data and have been involved in drafting the manuscript. NG has helped in the analysis and interpretation of data and has given final approval of the version to be published. All authors read and approved the final manuscript.

\section{Acknowledgements}

The financial assistance from the VIT University is gratefully acknowledged. We express our sincere thanks to Mrs. Hemavathy (NIMHANS) for helping with the electron microscopy. SSN wishes to acknowledge with thanks the receipt of associateship from the VIT University. Thanks are also due to Mr. A. B. Aditya Naga Sai Naidu for his help in the collection of the specimens.

\section{Author details}

${ }^{1}$ School of Bio Sciences and Technology (SBST), VIT University, Vellore, Tamil Nadu 632014, India. ${ }^{2}$ Electron Microscopy facility, Department of Neuropathology, National Institute of Mental Health and Neurosciences (NIMHANS), Bangalore 560029, India.

Received: 16 May 2014 Accepted: 4 September 2014

Published online: 12 December 2014

\section{References}

Adiyodi KG, Adiyodi RG (1970) Endocrine control of reproduction in Decapod Crustacea. Biol Rev 45:121-165

Aoto T, Kamiguchi Y, Hisano S (1974) Histological and ultrastructural studies on the Y-organ and mandibular organ of the freshwater prawn, Palaemon poucidens, with special reference to their relation with the molting cycle. J Fac Sci Hokkaido Univ Ser Vi Zool 19:295-306

Borst DW, Laufer H, Landau M, Chang E, Hertz W, Baker F, Schooley D (1987) Methyl farnesoate and its role in crustacean reproduction and development. Insect Biochem 17:1123-1127

Byard EH, Shivers R, Aiken DE (1975) The mandibular organ of the lobster, Homarus americanus. Cell Tissue Res 162:13-22

Gunawardene YI, Tobe SS, Bendena WG, Chow BK, Yagi KJ, Chan SM (2002) Function and cellular localization of farnesoic acid O-methyltransferase (FAMet) in the shrimp, Metapenaeus ensis. Eur J Biochem 269(14):3587-3595

Hinsch GW (1981) The mandibular organ of the female spider crab, Libinia emarginata, in immature, mature, and ovigerous crabs. J Morphol 168(2):181-187

Kappalli S, Supriya NT, Krishnakumar V, Anilkumar G, Chang ES (2012) Hemolymph ecdysteroid titers in a brachyuran crab (Uca triangularis) that concomitantly undergoes molting and reproduction. Zool Stud 51:966-976

Laufer H, Landau M, Homola E, Borst DW (1987) Methyl farnesoate: its site of synthesis and regulation of secretion in a juvenile crustacean. Insect Biochem 17(7):1129-1131

Laufer H, Biggers WJ, Ahl JS (1998) Stimulation of ovarian maturation in the crayfish Procambarus clarkii by methyl farnesoate. Gen Comp Endocrinol $111(2): 113-118$

Le Roux A (1968) Description d'organes mandibulaires nouveaux chez les crustacés Decapodés. C R Hebd Acad Sci Ser D Sci Nat 266:1414-1417

Nagaraju GPC (2011) Reproductive regulators in decapod crustaceans: an overview. J Exp Biol 214:3-16

Nagaraju GPC, Reddy PR, Reddy PS (2004) Mandibular organ: its relation to body weight, sex, molt and reproduction in the crab, Oziotelphusa senex senex. Aquaculture 232:603-612

Nagaraju GPC, Reddy PR, Reddy PS (2006) In vitro methyl farnesoate secretion by mandibular organs isolated from different molt and reproductive stages of the crab Oziotelphusa senex senex. Fisheries Sci 72(2):410-414

Nagaraju GPC, Rajitha B, Borst DW (2011) Molecular cloning and sequence of retinoid $x$ receptor in the green crab Carcinus maenas: a possible role in female reproduction. J Endocrinol 210:379-390

Sarika SN, Anilkumar G (2014) DNA binding domain of retinoid receptor gene (RXR) from a field crab inhabiting Indian peninsula. Ind J Biotech 13:52-56

Sudha K, Anilkumar G (2007) Elevated ecdysteroid titer and precocious molt and vitellogenesis induced by eyestalk ablation in the estuarine crab, Metopograpsus messor (Brachyura: Decapoda). J Crustacean Biol 27:304-308
Tamone SL, Chang ES (1993) Methyl farnesoate stimulates ecdysteroid secretion from crab y-organs in vitro. Gen Comp Endocrinol 89:425-432

Teal PE, Jones D, Jones G, Torto B, Nyasembe V, Borgemeister C, Alborn HT, Kaplan F, Boucias D, Lietze VU (2014) Identification of methyl farnesoate from the hemolymph of insects. J Nat Prod 77(2):402-405

Tiu SH, Hult EF, Yagi KJ, Tobe SS (2012) Farnesoic acid and methyl farnesoate production during lobster reproduction: possible functional correlation with retinoid x receptor expression. Gen Comp Endocrinol 175(2):259-269

Wang YH, LeBlanc GA (2009) Interactions of methyl farnesoate and related compounds with a crustacean retinoid $X$ receptor. Mol Cell Endocrinol 309:109-116

Yudin Al, Diener RA, Clark WH, Chang ES (1980) Mandibular gland of the blue crab, Callinectes sapidus. Biol Bull 159:760-772

doi:10.1186/s40555-014-0065-8

Cite this article as: Sarika et al.: Secretory activity of mandibular organ

fluctuates in response to reproductive season of the field crab

Paratelphusa sp. (Brachyura; Decapoda): an ultrastructural study.

Zoological Studies 2014 53:65.

\section{Submit your manuscript to a SpringerOpen ${ }^{\circ}$ journal and benefit from:}

- Convenient online submission

- Rigorous peer review

- Immediate publication on acceptance

- Open access: articles freely available online

- High visibility within the field

- Retaining the copyright to your article

Submit your next manuscript at $>$ springeropen.com 